\title{
RESPONSABILIDAD SOCIAL UNIVERSITARIA EN TIEMPOS DE PANDEMIA: MIRADA DESDE LA FUNCIÓN DOCENTE (UNIVERSIDAD DE ANTOFAGASTA - CHILE)
}

\author{
RESPONSABILIDADE SOCIAL DA UNIVERSIDADE EM TEMPOS DE PANDEMIA: \\ UM OLHAR A PARTIR DA FUNÇÃO DOCENTE (UNIVERSIDADE DE \\ ANTOFAGASTA - CHILE)
}
UNIVERSITY SOCIAL RESPONSIBILITY IN TIMES OF PANDEMIC: VIEW FROM THE TEACHING FUNCTION (UNIVERSITY OF ANTOFAGASTA - CHILE)

\author{
Oscar ROJAS ${ }^{1}$ \\ Marlenis MARTÍNEZ ${ }^{2}$ \\ Amely VIVAS ${ }^{3}$
}

RESUMEN: El presente artículo tiene como objetivo analizar la responsabilidad social universitaria en tiempos de pandemia, mirada desde la función docente en la promoción del autocuidado de los estudiantes. Para ello se utilizó un enfoque cuantitativo y diseño no experimental transeccional-descriptivo. Los participantes fueron 85 docentes de la carrera de pedagogía en educación básica de la Universidad de Antofagasta. Se aplicó muestreo probabilístico intencional y se utilizó cuestionario con escalamiento tipo Likert. Los resultados demostraron que los docentes manejan la responsabilidad social universitaria en tiempos de pandemia, sin embargo, necesitan fortalecer la promoción del autocuidado en los estudiantes y el manejo del conocimiento sobre pandemia con herramientas poderosas de cuidado, protección y resguardo para la vida. Los hallazgos encontrados permitieron concluir que los docentes deben promover estrategias de promoción del autocuidado, tales como el uso de podcast, vodcast, videos y cápsulas educativas que acceden a la comprensión de la pandemia COVID-19.

PALABRAS CLAVE: Responsabilidad social. Tiempo. Pandemia. Docente.

RESUMO: Este artigo tem como objetivo analisar a responsabilidade social universitária em tempos de pandemia, a partir do papel do professor na promoção do autocuidado do aluno. Para tanto, utilizou-se uma abordagem quantitativa e um desenho descritivo-transversal não experimental. Os participantes foram 85 professores da pedagogia da educação básica da

\footnotetext{
${ }^{1}$ Universidad Miguel de Cervantes (UMC), Santiago de Chile - Chile. Estudiante de doctorado en Educación. Postdoctorado en Finanzas. ORCID: https://orcid.org/0000-0002-6739-5559. E-mail: oscar.rojas@umcervantesecontinua.cl

2 Universidad Miguel de Cervantes (UMC), Santiago de Chile - Chile. Postdoctorado em Gestión del conocimiento (Universidad Pedagógica Experimental Libertador). Doctorado en Ciencias de la Educación (Universidad Fermín Toro). Magíster en Educación, Mención Gerencia Educacional (Universidad Pedagógica Experimental Libertador-Instituto Pedagógico de Barquisimeto). ORCID. https://orcid.org/0000-0002-5926599X.E-mail: marlenism3@gmail.com

${ }^{3}$ Universidad Miguel de Cervantes (UMC), Santiago de Chile. Post Doctor en Estudios Libres, Doctora en Ciencias de la Educación, Maestría en Planificación Educativa, Especialista en Evaluación Educacional. ORCID: https://orcid.org/0000-0002-5791-8619-mail: amelydvivase@gmail.com
}

RIAEE - Revista Ibero-Americana de Estudos em Educação, Araraquara, v. 16, n. 2, p. 424-439, abr./jun. 2021. e-ISSN: 1982-5587 DOI: https://doi.org/10.21723/riaee.v16i2.14707 
Universidade de Antofagasta. Foi aplicada amostra probabilística intencional e questionário do tipo Likert. Os resultados mostraram que os professores lidam com a responsabilidade social universitária em tempos de pandemia, no entanto, eles precisam fortalecer a promoção do autocuidado dos alunos e a gestão do conhecimento sobre a pandemia com ferramentas poderosas de cuidado, proteção e salvaguarda da vida. Os resultados encontrados permitiram concluir que os professores devem promover estratégias de promoção do autocuidado, como o uso de podcasts, vodcasts, vídeos e cápsulas educativas que acessem a compreensão da pandemia COVID-19.

PALAVRAS-CHAVE: Responsabilidade social. Tempo. Pandemia. Professor.

ABSTRACT: The This article aims to analyze university social responsibility in times of pandemic, viewed from the teaching role in promoting student self-care. For this, a quantitative approach and a non-experimental transectional-descriptive design were used. The participants were 85 teachers from the basic education pedagogy at the University of Antofagasta. Intentional probability sampling was applied and a Likert-type questionnaire was used. The results showed that teachers handle university social responsibility in times of pandemic, however, they need to strengthen the promotion of self-care in students and the management of knowledge about pandemic with powerful tools for care, protection and safeguarding of life. The findings found allowed to conclude that teachers should promote self-care promotion strategies, such as the use of podcasts, vodcasts, videos and educational capsules that access the understanding of the COVID-19 pandemic.

KEYWORDS: Social responsibility. Time. Pandemic. Teacher.

\section{Introducción}

La educación está recibiendo influencias de distintas organizaciones, instituciones y otros entes, con el propósito de proporcionar respuestas a las exigencias de la globalización. La misma esta reforzada e impulsada estrechamente por los avances tecnológicos en las comunicaciones, el transporte y los medios de transmisión de información (GARBANZO, 2015).

Se trata de avanzar, desde el individuo, hacia contextos de responsabilidad, reflexión y producción, sementadas en la edificación colectiva de prácticas epistemológicas de pensamiento en escenarios multireferenciales, enmarcados en la formación de una ética de razón que posibilite articular los distintos saberes en forma no reduccionista. Asimismo, la promoción de la cosmovisión de la humanidad en articulación con nuevas ideas, diálogos y vinculadas con la responsabilidad de crear un futuro más solidario.

Es así como en los países de Iberoamérica, tal como se presentó en el Congreso Iberoamericano de Educación en el año 2010, se visualizan las diferentes experiencias de participación en América Latina, asumiendo que la colaboración va más allá de las aulas de 
clase, en otras palabras, el espacio social permitirá desarrollar situaciones democráticas participativas (ARANCIBIA; QUINTERO, 2010). Sin embargo, a pesar de dichas evidencias, la contribución social en la educación constituye un campo en construcción colmado de prácticas que no alcanzan de normalizarse y acceder a complementar constructos teóricos para analizar la participación social en la educación como un fenómeno complejo.

En efecto, la educación, como instrumento para alcanzar sociedades con una democracia más firme requiere que la sociedad busque hacer más equivalentes o iguales en la repartición de bienes, en la cual exista una conexión con lo social, educativo, político, económico, se superen los obstáculos y se afiancen comportamientos proactivos, efectivos y eficaces en el desarrollo humano.

Es por ello que, grandes investigadores, en diversos países del mundo, han disertado y realizado estudios sobre la responsabilidad y en cada una de ellas, en mayor o menor proporción, planteando líneas o estrategias en función de dar cumplimiento a las mismas (SEVERINO; MEDINA; PUJOL, 2018). Sin embargo, es importante acotar, que posiblemente, lo que más afecta en aplicar la responsabilidad en cualquier empresa o institución, desde el punto de vista interno y externo, lo representa los factores económicos, sociales, culturales, ambientales, políticos, tecnológicos, entre otros.

La génesis del concepto de RS no se encuentra precisada exactamente, es decir, los estudiosos, investigadores y expertos no han acordado bien su principio, específicamente lo relacionado a cuando y donde fue originado (PÉREZ; ESPINOZA; PERALTA, 2016). Esto puede deberse a las diferentes corrientes de conocimientos de fuentes económicas, políticas, sociales o filosóficas que han influido en el progreso y exposición sobre este tema en el devenir de la historia. Sin embrago, la responsabilidad social existe un gran número de estudiosos que consideran que la misma se origina alrededor del ámbito empresarial.

Por lo tanto, lo más cercano a la definición de RS lo representa la manera de formar negocios donde se tome en cuenta la relevancia social, ambiental, económica y política de una institución; integrando principios y valores en los trabajadores, comunidades y el entorno en cual se encuentra inmersa (MONTAÑEZ; GUTIÉRREZ, 2015).

Por lo tanto, la Responsabilidad Social (RS), forma una práctica fundamental en la gestión del procedimiento moral y diáfano en cualquier institución (GÓMEZ; ALVARADO; PUJOLS, 2018). Debe ser un principio obligatorio para las organizaciones educativas, bien sea de índole pública o privada, razón por la cual requiere de procesos complejos de incorporar todo lo relacionado a los ámbitos académicos, convivencia, autocuidado, entre otros.

RIAEE - Revista Ibero-Americana de Estudos em Educação, Araraquara, v. 16, n. 2, p. 424-439, abr./jun. 2021. e-ISSN: 1982-5587 
La RS está de boga, no sólo en el mundo empresarial, sino también a nivel universitario. Es un término bastante novedoso y un tanto complejo de delimitar, razón por la cual involucra muchos actores de diversos horizontes e intereses. Tuvo sus inicios en las empresas, la misma comienza a expandirse hacia los años sesenta, específicamente en ciudades inglesas como Estados Unidos, Canadá o Reino Unido. La RS empresarial "es como la forma de hacer negocios generando impactos positivos para la sociedad y el medio" (SEPÚLVEDA; URRUTIA, 2013, p. 9). En otras palabras, es la manera como una empresa se proyecta en la sociedad y el entorno en la cual se encuentra anclada y, por ende, representa la suposición moral o filosófico a través de un gerente, compañía, institución o sujeto que tiene la responsabilidad de mejorar el destino de la humanidad.

En este orden de ideas, la Responsabilidad Social Universitaria (RSU) trasciende en su labor, en sus reflexiones y diálogos académicos en función de vincular la comunidad con el contexto en el cual se encuentra anclada (HERNÁNDEZ; MORA, 2017). Es decir, es responsabilidad de la universidad de alcanzar o mediar el bienestar de todos sus integrantes y ser extendidos a la comunidad-sociedad. Asimismo, mantiene una definición más extendida, razón por la cual debe abocarse o apropiarse de la comunidad universitaria como una manera de emprender su labor de gestión y excelencia educativa (ALDEANUEVA; ARRABAL, 2018).

En efecto, la RSU tiene la capacidad de promover y situar la praxis de un conjunto de principios y valores éticos-morales a través de los procesos de gestión, docencia, investigación y extensión; asumiendo responsabilidades directas en su organización, en el contexto, la región y país en la cual se encuentra (VALLAEYS; ÁLVAREZ, 2019). La RSU representa disímiles elementos que son relevantes, entre ellos: el compromiso cívico, estudiantes activos, voluntariado, el fomento de la colaboración ambiental, el fortalecimiento de las comunidades, la optimización del proceso enseñanza-aprendizaje, la convivencia, al autocuidado de los estudiantes u otros aspectos importantes en el desarrollo de la verdadera responsabilidad. Además, simboliza la capacidad de fomentar valores por medio de la gestión responsable, indagación y participación de todos los entes (GÓMEZ; ALVARADO; PUJOLS, 2018).

En consecuencia, la RSU desde la función docente, el profesor cobra bastante significado en el desarrollo de las actividades de docencia, investigación, extensión y gestión. Específicamente, la docencia concierne con la utilización de transmisión de conocimientos y metodologías de enseñanza a los estudiantes en el contexto universitario. (BUENESTADO, 2019). En otras palabras, la docencia no se fundamente solamente en estar en un aula de clase 
con estudiantes, sino que debe extenderse más allá del aula en la cual involucre activamente a los estudiantes en otras tareas que se encuentren en el entorno en cual se desenvuelve.

De igual manera, la función docente, está relacionado con el contexto histórico, ambiental, social, de salud, del autocuidado y de cada una de las actividades que se llevan a cabo en la universidad, dentro y fuera de ella; cumpliendo diversos roles que lo induce a ser investigadores datos y gestores del aprendizaje de los estudiantes.

En efecto, la función docente, en tiempos de pandemia, debe estar enfocado hacia las acciones que debe guiar en la promoción de la salud de los estudiantes, es decir, desde los contextos de la virtualidad generar actividades que conduzcan al autocuidado de los alumnos, familiares, entre otros. La RSU, se presenta como una nueva manera de accionar y de comportarse de manera fluida y directa del docente en relación con el ámbito académico, en estos tiempos de crisis sanitaria. Igualmente, el compromiso de los profesionales de la docencia, en estos momentos, debe situarse hacia la protección de la sociedad como aspecto dominante e imperativo ético en la labor del docente (GAETE, 2020).

Efectivamente, la RSU, focalizada desde la mirada de la función docente viene dada porque se hace necesario transformar el pensamiento de los estudiantes en función de hacer cara a la creciente complejidad, a la rapidez de las transformaciones y a la poca proyección que determina al mundo. Por lo tanto, se amerita reconstruir el conocimiento, lanzando por tierra estructuras esquemáticas antiguas e ir uniendo nuevos eventos de pensamientos humildes, sinceros, críticos, participativos y cuidados de manera integral de la salud física y mental de los estudiantes en escenarios transitorios.

Partiendo de lo antes expuesto y tomando en cuenta los últimos acontecimientos a nivel internacional en cuanto a la génesis y revolución relacionada con la Pandemia COVID19, que trajo consigo transformaciones, cambios de conducta, estilos de vida diferentes en cada uno de los individuos y, en especial en el funcionamiento de las universidades. De allí que, el afrontamiento de esta pandemia está colocando a prueba la capacidad de acomodo de manera propia y colectiva en la vida de las personas; a la vez las cuantiosas transformaciones por esta crisis y su impacto social en la promoción de comportamientos ante estos nuevos escenarios (ESPADA; ORGILÉS; PIQUERAS; MORALES, 2020).

Por lo tanto, la RSU en tiempos de crisis o pandemia, la cual vino a modificar abruptamente todos los escenarios a los cuales se estaba acostumbrado (CEBALLOS, 2020). Algunas instituciones, en especial en el ámbito de la Universidad, la misma ha cobrado mayor relevancia e impacto, razón por la cual se han tenido que valerse de muchas acciones, estrategias, técnicas y herramientas tecnológicas para continuar con las funciones de docencia, 
investigación y extensión. Dichas acciones se llevan a cabo para proteger las remuneraciones de sus profesores, la continuidad en la formación de los estudiantes, la investigación y extensión de todas sus actividades, es decir, es el momento de actuar rápido y fortalecer la sostenibilidad y seguir adelante (RAMÍREZ, 2020).

De allí que, las Universidades de Chile, no escapan a la situación planteada, razón por la cual la RSU cobra mayor auge para poder sobrellevar y funcionar de la manera más eficiente posible en el proceso educativo; considerándose un elemento fundamental en la formación de los estudiantes, a objeto de fortalecer todos los procesos relacionados el compromiso de los docentes, capacidad para dar respuesta a los problemas sociales, ambientales, económicos y sociales dentro y fuera de la institución aplicando, para ello, la función docencia universitaria ante la crisis sanitaria que vive el país por el COVID-19.

En este orden de ideas, la interrupción de las clases presenciales en todo el territorio chileno en la cual aceleradamente se implementó la educación a distancia o remota (BELLEI; MUÑOZ, 2020), representó un reto para los educadores de las universidades, especialmente la Universidad de Antofagasta, Región Antofagasta en las carreras de pedagogía de la educación, donde los docentes se han visto en la necesidad de girar sus actividades desde otra visión. Es decir, los profesores tienen que efectuar cursos en línea de los contenidos de las asignaturas, sin mayor preparación, formación, diseño o realidad (CEA et al., 2020). Todo esto ha generado una serien de situaciones que el profesor ha tenido que enfrentar y dar continuidad al proceso enseñanza-aprendizaje.

De acuerdo con Navarro del Instituto de Ciencias de la Educación de la Universidad de O’Higgins, citado por Sepúlveda (2020), plantea que los procesos de enseñanza y aprendizaje realizados en las claves virtuales son externos a los aspectos afectuosos y emocional de los estudiantes. En este proceso de transición que se está viviendo, muchos estudiantes están pasando por momentos de angustias e intranquilidad por diversas razones, entre ellas, angustia por el contagio, riesgos de contagios con sus familiares, el encierro en los hogares y el autocuidado.

El autocuidado representa un aspecto relevante y significativo en tiempos de pandemia, razón por la cual los profesores, por medio de la utilización de las herramientas tecnológicas y la responsabilidad social, pueden generar la prevención y promover el cuidado de los estudiantes y conseguir escenarios de bienestar y la promoción de una adecuada salud (CHILE, 2020). El autocuidado tiene su origen en la salud pública, especialmente a partir de la praxis de la enfermería. El mismo se define como aquellas acciones que efectúan los sujetos con la finalidad de promover la salud, enfrentar padecimientos, malestares y 
sufrimientos ante cualquier situación de pandemias, enfermedades o catástrofes (OLTRA, 2013).

En efecto, desde la función docente se deben generar acciones preventivas del autocuidado a los estudiantes frente a la pandemia del COVID-19, donde en una interacción contante-participativa virtual existan las medidas de prevención, tanto afectivas, emocionales y del cuidado general de sus familiares, amigos y comunidad.

Partiendo de lo antes expuesto, el presente artículo tiene como objetivo analizar la responsabilidad social universitaria en tiempos de pandemia, mirada desde la función docente específicamente en la promoción del autocuidado de los estudiantes; generando acciones o actividades que contribuyan con el bienestar, el autocuidado frente a la crisis sanitaria y el compromiso responsable hacia ellos.

El estudio reviste importancia porque indagar en este tema, en tiempos transitorios, conlleva a que los profesionales de la docencia deben tener la oportunidad de aplicar la responsabilidad social universitaria con los estudiantes en el autocuidado y sea extrapolada a sus familiares en el cuidado de la salud, bienestar y la prevención ante una pandemia que ha traído desaciertos, incertidumbre y poca profundidad en el tratamiento y medicación para su propagación. Asimismo, se genera la siguiente interrogante: ¿Cuál es la responsabilidad social universitaria en tiempos de pandemia, mirada desde la función docente específicamente en la promoción del autocuidado de los estudiantes de las carreras de pedagogía en educación de la Universidad de Antofagasta-Chile?

\section{Metodología}

El estudio se enmarcó en el enfoque cuantitativo, el cual refleja la amplitud y profundidad del conocimiento investigativo; buscando medir todos los elementos que se encuentran en el objeto de estudio, con la finalidad de describirlos, analizarlos e interpretarlos (ARIAS, 2016). En otras palabras, el presente artículo está representado por la responsabilidad social universitaria en tiempos de pandemia y la función docente, se escribirán los elementos relacionados con la variable e indicadores de estudio.

El diseño del estudio se enmarcó en una investigación no experimental transeccionaldescriptivo porque se observa el contexto en el cual ocurren los hechos sin tener ningún tipo de manipulación de las variables y realizando el abordaje una determinada población en un grupo de personas (HERNÁNDEZ; MENDOZA, 2018). 
Los participantes constituidos por 85 docentes de las carreras de pedagogía en educación básica de la Universidad de Antofagasta, ubicada en la comuna de Antofagasta, Región de Antofagasta-Chile. Asimismo, se utilizó un muestreo no probabilístico intencional porque dependió de la intención del investigador de indagar la responsabilidad social en tiempos de pandemia desde la función docente en el autocuidado de los estudiantes de la mencionada carrera.

En efecto, el confinamiento de los profesionales de la docencia ha generado factores negativos producto del encierro y la lucha a diario a través de la implementación de herramienta de apoyo psicoemocional y resolución de conflictos, tanto personales como la mediación de problemas por los educandos. Es importante acotar que la muestra seleccionada tiene características muy similares, razón por la cual los docentes imparten clases en el área educativa y todos se encuentran en confinamiento.

El instrumento empleado fue un cuestionario con escalamiento tipo Likert, donde se plantearon afirmaciones y se les pidió a los sujetos que expresarán su reacción, seleccionando una de las tres categorías de respuestas: De acuerdo (DA, 3), Neutro (N, 2) y En desacuerdo (ED, 1) (HERNÁNDEZ; FERNÁNDEZ; BAPTISTA, 2014), con un total de 9 preguntas en la cual las ponderaciones altas muestran una actitud más positiva a las respuestas emitidas en las categorías. La aplicación del mismo fue en el mes de abril de 2020.

La validación del instrumento se efectuó a través de los análisis de contenido por medio de tres expertos en metodología, estadística y especialista en responsabilidad social universitaria, los mismos concordaron que el cuestionario se encontraba a acorde al estudio de las variables. Seguidamente, se aplicó una prueba piloto a 20 docentes, vía correo electrónico, que no pertenecían a la muestra de estudio y tenían las mismas características. Asimismo, se usó el coeficiente de alfa Cronbach, obteniendo un valor de 0,93 considerándose confiable.

Tomando en cuenta el confinamiento de los docentes de la Universidad de Antofagasta, se envió, vía correo electrónico, una convocatoria para dos reuniones virtuales a través de la plataforma zoom, la primera de ellas fue para informar lo relacionado con la justificación, los objetivos y la intención de la investigación, al final de la cual la muestra seleccionada se comprometió a la participación en la aplicación del instrumento.

En la segunda reunión se pautó el consentimiento, la ética y las instrucciones para responder al instrumento enviado por medio de la plataforma referida; acordadas las pautas los docentes, voluntariamente, respondieron a los ítems. Igualmente, se procedió a la elaboración de tabla y de gráfico, donde se realizaron el análisis de los ítems por indicadores: responsabilidad social universitaria, Pandemia COVID-19 y el autocuidado. 


\section{Resultados}

Los resultados obtenidos en el indicador responsabilidad social universitaria, se pueden visualizar en la tabla 1 , de los cuales el 80,39\% de los docentes consideran tener una actitud favorable con respecto al manejo de la responsabilidad social universitaria, desde la función docente en tiempos de pandemia, gestionan la enseñanza de valores éticos y morales para la protección personal-familiar y consideran que la responsabilidad social universitaria le permite un acercamiento socioafectivo con los estudiantes, Sin embargo, el 11,37\% manifestaron una actitud desfavorable hacia la responsabilidad social. En efecto, la responsabilidad social desde la función docente debe darse de manera holística y concatenada en la promoción social de nociones moralistas en la transmisión de conocimientos responsables (OJEDA, 2013).

En lo que respecta al indicador pandemia COVID-19, en la tabla 1 se puede observar que el promedio de respuestas se ubicó en un $83,5 \%$ de actitud desfavorable en cuanto a la motivación de los estudiantes en la indagación sobre la pandemia COVID-19, además de mantener, permanentemente, informado al estudiante sobre la pandemia y desde la función docente la promoción de conocimientos y participación de actividades inherentes a las medidas preventivas. El 9,4\% mostró una actitud favorable a las actividades antes descritas.

La Organización Mundial de la Salud, ha implementado acciones en iniciar el autocuidado y el distanciamiento social entre los individuos; buscando alternativas de prevención para que la pandemia pueda propagarse o en su defecto disminuir los contagios. (MONTENEGRO, 2020).

De igual manera, en la tabla 1 se puede precisar el indicador de autocuidado cuyo promedio de respuesta se ubicó en el 90,59 \% de actitud desfavorable hacia la articulación de la promoción del autocuidado de los estudiantes desde las clases virtuales, la transmisión de saberes responsables en el cuidado y protección en lo físico y mental a la pandemia COVID19 y la aplicación de acciones en la construcción de respuestas exitosas para atender los retos que implica el autocuidado de los estudiantes en tiempo de pandemia. El 4,3 por ciento demostraron un ambiente favorable hacia las acciones antes mencionadas. Este resultado se contradice con el planteamiento de REYES (2019) cuando expone que el autocuidado representa acciones que indicen a una buena práctica de cuidado en el individuo y por ende generar un mejor bienestar y calidad de vida. Estos resultados fueron representados en el gráfico 1, donde se aprecian los promedios obtenidos en las respuestas proporcionadas por los sujetos encunetados de acuerdo a los indicadores indagados.

RIAEE - Revista Ibero-Americana de Estudos em Educação, Araraquara, v. 16, n. 2, p. 424-439, abr./jun. 2021. e-ISSN: 1982-5587 
Tabla 1 - Distribución del promedio de respuesta por indicador

\begin{tabular}{|c|c|c|c|}
\hline Indicador & De acuerdo (3) & Neutro (2) & En Desacuerdo (1) \\
\hline $\begin{array}{c}\text { Responsabilidad Social } \\
\text { Universitaria }\end{array}$ & 80,39 & 8,24 & 11,37 \\
\hline COVID-19 & 9,4 & 7,06 & 83,5 \\
\hline Autocuidado & 4,3 & 5,1 & 90,59 \\
\hline
\end{tabular}

Fuente: Elaboración propia

\section{Discusión}

Los resultados obtenidos, se puede evidenciar que los docentes de la carrera de pedagogía en educación básica de la Universidad de Antofagasta mantienen una actitud bastante favorable hacia el reconocimiento de la RSU, en la cual la mayoría reconoce la importancia y relevancia de la misma en las actividades educativas en tiempos de pandemia. Esto es bastante significativo, para el presente estudio, porque los educadores, a pesar de estar en situaciones de confinamiento en sus hogares muestran actitudes positivas hacía el desarrollo de la RSU. Esto es avalado con el estudio de Gaete (2017), cuando refiere que la RSU permite la gestión y la resolución de problemas educativos y sociales con efectividad y, así poder equilibrar sus compromisos responsables con los estudiantes y las familias.

De igual manera, la indagación de Aldeanueva y Arrabal (2018) la RSU, es primordial para el desarrollo de las comunidades en las cuales se encuentran ancladas y de allí desarrollar actividades que vayan en beneficio de los estudiantes. Es este aspecto es relevante en tiempos de pandemia porque los docentes deben avocarse a la emergencia sanitaria que se está viviendo y poder mediar situaciones de colaboración, de manera responsable, a los estudiantes y demás integrantes de la familia. Igualmente, la investigación realizada por Barajas, Benítez y Ramírez (2020) la RSU representa una toma de conciencia en la mediación de problemas sociales avaladas por principios éticos en función de garantizar la organización, trabajo eficiente, el bienestar social y las acciones hacia el bien común de todos.

Por otra parte, los datos obtenidos en el indicador de pandemia COVID-19, se observa que la gran mayoría de los docentes tienen una actitud muy desfavorable en cuanto a la motivación, información y promoción de la pandemia; evidenciándose el poco interés de los 
profesionales de la docencia de impulsar estrategias investigativas relacionada con la crisis sanitaria que se vive actualmente y que está afectando a un sin número de estudiantes; bien sea por el acercamiento hacia el conocimiento de los efectos, causas y consecuencias de la pandemia sobre las personas y el entorno en cual se desenvuelve.

En efecto, el estudio de Moya (2020), plantea que los riesgos que se corren con la pandemia COVID-19 son bastantes fuertes y drásticos, para lo cual se requiere sujetar, aminorar y mermar los impactos que esta genera; para ello se requiere la gestión integrada de todos los individuos, especialmente el ámbito educativo, en conducir acciones que contribuyan con la información y manejo de la enfermedad.

El COVID-19, es un asunto de salud y responsabilidad social reseña que la RSU cobra mayor realce porque se debe tener empatía y pensar en el cuidado del otro y este aspecto es primordial en el nivel educativo, son elementos que se debe enseñar y promocionar en la virtualidad (VERA, 2020). Igualmente, son relevantes los aportes efectuados por Cortés (2020) indicando que, frente al brote de la pandemia, que cada día se incrementa más en las vidas de los ciudadanos en todo el mundo, es fundamental la disponibilidad de información precisa, pertinente y veraz de los efectos, causas y consecuencias de la pandemia COVID-19. Es decir, manejar un conocimiento adecuado de la pandemia con todos los habitantes y, especialmente en los estudiantes universitarios representa una herramienta poderosa de cuidar, proteger y resguardar la vida para hoy y el futuro.

Por último, el indicador relacionado con el autocuidado, en los hallazgos encontrados se evidencia que los docentes carecen de la promoción y difusión del cuidado a través de las clases virtuales; generando, posiblemente, darles relevancia a los aspectos socioafectivos en la prevención de la salud integral de los estudiantes. Asimismo, Vera (2020), expone que la práctica del autocuidado como una manera efectiva de generar ambientes educativos para resguardar la salud; desarrollando habilidades y destrezas del cuidado emocional y físico del sujeto.

De igual manera, Escobar y Pico (2013) plantean que el autocuidado depende, en cierta medida, de la motivación que el estudiante universitario reciba de sus profesores en función de valorar, de manera permanente, el cuidado de su salud y bienestar que reciban en el contexto en cual se desenvuelven. Significa que las acciones que genere el docente conducirán al bienestar de los educandos. También, Escobar, Franco y Duque (2011) hacen alusión que los profesores universitarios les incumbe asumir el autocuidado de los estudiantes como una acción cotidiana el quehacer educativo, es decir, les compete planificar actividades que vayan en beneficio del bienestar de sus propias vivencias.

RIAEE - Revista Ibero-Americana de Estudos em Educação, Araraquara, v. 16, n. 2, p. 424-439, abr./jun. 2021. e-ISSN: 1982-5587 
Los hallazgos encontrados inducen a concluir que los docentes de la carrera de pedagogía de educación Básica de la Universidad de Antofagasta manejan el conocimiento de la RSU, sin embargo, ameritan consolidar estrategias de promoción del autocuidado, tales como el uso de podcast, vodcast, videos, cápsulas educativas, entre otras que acceden al conocimiento de la pandemia COVID-19 y la manera de resguardar la integridad física, emocional y mental de ellos y pueda set extrapola a sus familiares. Es importante, darle a conocer los estudiantes el cuidado como ser, como individuo frente a una pandemia que se considera incierta de quedarse en las vidas humanas o poder erradicarse en los próximos años.

Con los resultados obtenidos, en este estudio, no son determinantes se sugiere continuar con nuevos estudios, con la finalidad de profundizar más sobre la responsabilidad social del docente y las medidas sanitarias que se deben impartir en tiempos transitorios y de emergencia; se está frente una pandemia que desconoce un tratamiento o cura efectiva, por lo tanto, es un tema largo y minucioso de indagar. Sería importante que otras universidades tomen en cuenta el análisis de esta investigación y pueda ser extensivo en otros espacios educativos.

\section{REFERENCIAS}

ALDEANUEVA, I.; ARRABAL, G. La comunicación y medición de la responsabilidad social universitaria: redes sociales y propuesta de indicadores. Revista Digital de Investigación en Docencia Universitaria, v. 12, n. 1, p. 121-136, 2018. DOI: http://dx.doi.org/10.19083/ridu.12.626

ARANCIBIA, M.; QUINTERO, S. Metas educativas 2021. Participación social en educación: una construcción común. 2010. Disponible: http://www.adeepra.org.ar/congresos/Congreso\%20IBEROAMERICANO/METAS2021/RLE 2120_Arancibia.pdf. Acceso: 10 dic. 2020.

ARIAS, F. EI proyecto de investigación: guía para su elaboración. 7. ed. Caracas: Episteme. 2016. ISBN: 980-07-3868-1.

BARAJAS, J.; BENÍTEZ, M.; RAMÍREZ, R. Responsabilidad social del estudiante en una universidad pública de México. Revista Venezolana de Gerencia, v. 25, n. 91, 2020.

Disponible:

https://www.produccioncientificaluz.org/index.php/rvg/article/view/33207/34873. Acceso: 10 dic. 2020 .

BELLEI, C.; MUÑOZ, G. Educación a distancia y vuelta al colegio: la reinvención de la comunidad escolar. Ciper Académico (Columna de opinión), 2020. Disponible: https://ciperchile.cl/2020/07/21/educacion-a-distancia-y-vuelta-al-colegio-la-reinvencion-dela-comunidad-escolar/. Acceso: 10 dic. 2020. 
BUENESTADO, M. La formación docente del profesorado universitario. El caso de la Universidad de Córdoba (Diagnóstico y Diseño Normativo). Universidad de Córdova, Córdova, 2019. Disponible:

https://helvia.uco.es/xmlui/bitstream/handle/10396/18856/2019000001931.pdf?sequence=1\&i sAllowed=y. Acceso: 10 dic. 2020

CEA, F. et al. Educación online de emergencia: Hablando a pantallas en negro. Ciper académico (Columna de opinión), 2020. Disponible:

https://www.ciperchile.cl/2020/06/08/educacion-online-de-emergencia-hablando-a-pantallasen-negro/. Acceso: 10 dic. 2020.

CEBALLOS, P. Responsabilidad social empresarial en tiempos de crisis. La discusión cl. Disponible: http://www.ladiscusion.cl/responsabilidad-social-empresarial-en-tiempos-decrisis/. Acceso: 10 dic. 2020.

CHILE. MINISTERIO DE SALUD. La importancia del autocuidado en tiempos de pandemia. Artículo de opinión, 4 jun. 2020. Disponible: https://www.saludohiggins.cl/laimportancia-del-autocuidado-en-tiempos-de-pandemia/. Acceso: 10 dic. 2020.

CORTÉS, M. Coronavirus como amenaza a la salud pública. Rev. méd. Chile, Santiago, v. 148, n. 1, ene. 2020. DOI: http://dx.doi.org/10.4067/S0034-98872020000100124

ESCOBAR, P.; FRANCO, Z.; DUQUE, J. El autocuidado: un compromiso de la formación integral en educación superior. Hacia la Promoción de la Salud., v. 16, n. 2, p. 132-146, jul./dic. 2011. ISSN 0121-7577.

ESCOBAR. M.; PICO, M. Autocuidado de la salud en jóvenes universitarios, Manizales, 2010-2011. Rev. Fac. Nac. Salud Pública, v. 31, n. 2, p. 178-186, 2013. Disponible: http://www.scielo.org.co/pdf/rfnsp/v31n2/v31n2a03.pdf. Acceso: 10 dic. 2020.

ESPADA, J.; ORGILÉS, M.; PIQUERAS, J.; MORALES, A. Las buenas prácticas en la atención psicológica infanto-juvenil ante el Covid-19. Clínica y Salud: Investigación Empírica en psicología, v. 31, n. 2, p. 109-113, 2020. e-ISSN: 2174-0550. DOI: https://doi.org/10.5093/clysa2020a14

FERNÁNDEZ, B. Docencia en tiempos de pandemia: un llamado a una mirada sistémica. La Tercera, Artículo de opinión, 30 abr. 2020. Disponible: https:/www.latercera.com/quepasa/noticia/docencia-en-tiempos-de-pandemia-un-llamado-a-una-miradasistemica/SSC7ITGVLNCMTCMKMIEXQ4AV24/. Acceso: 10 dic. 2020.

GAETE, R. Conciliación trabajo-familia y responsabilidad social universitaria: Experiencias de mujeres en cargos directivos en universidades chilenas. Revista Digital de Investigación en Docencia Universitaria, v. 12, n. 1, p. 81-100, 2018. DOI:

http://dx.doi.org/10.19083/ridu.12.615

GAETE, R. Dirección por valores y responsabilidad social en universidades estatales chilenas. Revista Digital de Investigación en Docencia Universitaria, v. 14, n. 1, p. 1-14, ene./jun. 2020. DOI: http://dx.doi.org/10.19083/ridu.2020.1073 
GARBANZO, G. Desarrollo organizacional y los procesos de cambio en las instituciones educativas, un reto de la gestión de la educación. Revista Educación, v. 40, n. 1, p. 67-87, 2015. Universidad de Costa Rica. DOI: http://dx.doi.org/10.15517/revedu.v40i1.22534

GÓMEZ, L.; ALVARADO, Y.; PUJOLS, A. Practicando la responsabilidad social Universitaria en el Caribe: perspectivas de los públicos internos. Revista Digital de Investigación en Docencia Universitaria, v. 12, n. 1, p. 96-118, 2018. DOI: http://dx.doi.org/10.19083/ridu.12.714

HERNÁNDEZ, I.; MORA, J. Universidad y empresa: un binomio de responsabilidad social en el siglo XXI. Tendencias: Revista de la Facultad de Ciencias Económicas y Administrativas de Nariño, v. XVIII, n. 1, p. 145-158, ene./jun. 2017. Disponible: https://revistas.udenar.edu.co/index.php/rtend/article/view/3159/3725.

HERNÁNDEZ, R.; MENDOZA, C. Metodología de la investigación. Las rutas cuantitativas, cualitativas y mixta. México: Mc Graw Hill Education. 2018.

HERNÁNDEZ, S.; FERNÁNDEZ, C.; BAPTISTA, P. Metodología de la investigación. 5. ed. México: McGraw Hill, 2014. ISBN: 978-607-15-0291-9.

MONTAÑEZ, G.; GUTIÉRREZ, S. La responsabilidad social empresarial desde el enfoque de los grupos de interés. 1. ed. México: Cenid Editorial, 2015. ISBN: 978-6078435-11-1.

MONTENEGRO, D. Uso de tecnologías en el lugar de atención para el manejo de la pandemia por COVID-19 en Colombia. Rev. Panam. Salud Publica, v. 44, e97, 2020. DOI: https://doi.org/10.26633/RPSP.2020.97

MORALES, A.; ZACATENCO, J.; LUNA, M.; GARCÍA, R.; HIDALGO, C. Acceso y actitud del uso de Internet entre jóvenes de educación universitaria. Revista Digital de Investigación en Docencia Universitaria, v. 1 4, n. 1, ene./jun. 2020. ISSN: 2223-2516. DOI: https://doi.org/10.19083/ridu.2020.1174

MOYA, N. Responsabilidad y riesgos: conceptos claves en el debate ético generado por la COVID-19. Medisur, v. 18, n. 3, 2020. ISSN 1727-897X. Disponible:

http://scielo.sld.cu/scielo.php?script=sci_arttext\&pid=S1727-

897X2020000300512\&lng=es\&nrm=iso\&tlng=es. Acceso: 10 dic. 2020.

OJEDA, J. Responsabilidad social, construcción de un concepto desde la percepción del docente universitario. TEACS, año 5, n. 12, ene./jun. 2013. ISSN: 1856-9773.

OLTRA, S. El autocuidado, una responsabilidad ética. Bioética. Rev GPU, v. 9, n. 1, p. 8590, 2013. Disponible: https://medicina.udd.cl/centro-bioetica/files/2013/09/SANDRAOLTRA-El_autocuidado.pdf. Acceso: 10 dic. 2020.

PÉREZ, M.; ESPINOZA, C.; PERALTA, B. La responsabilidad social empresarial y su enfoque ambiental: una visión sostenible a futuro. Revista Universidad y Sociedad, v. 8, n. 3, p. 169-178, 2016. ISSN: 2218-3620. 
RAMÍREZ, J. Ética y responsabilidad social empresarial en tiempos de pandemia. Centro Sostenibilidad e Innovación Social (Centrum Think), 2020. Disponible:

https://centrumthink.pucp.edu.pe/etica-y-responsabilidad-social-empresarial-en-tiempos-depandemia. Acceso: 10 dic. 2020.

REYES, G. Factores que inciden en las prácticas de autocuidado en los uniformados de la dirección nacional de escuelas de la policía nacional: una mirada cualitativa. 2019. Tesis (Maestría) - Pontificia Universidad Javeriana, Bogotá, 2019. Disponible: https://repository.javeriana.edu.co/bitstream/handle/10554/43453/TESIS\%20AUTOCUIDAD O.pdf?sequence=1\&isAllowed=y. Acceso: 10 dic. 2020.

\section{SEPÚLVEDA, K.; URRUTIA, U. Responsabilidad social en la educación secundaria.} 2013. Disponible:

http://repobib.ubiobio.cl/jspui/bitstream/123456789/944/1/Sepulveda $\% 20$ Vasquez $\% 2 C \% 20 \mathrm{~K}$ aren.pdf. Acceso: 10 dic. 2020.

SEVERINO, P.; MEDINA, A.; PUJOL, L. Responsabilidad social en escuelas de educación primaria en Chile: tensiones y desafíos. Revista Encuentros, Universidad Autónoma del Caribe, v. 16, n. 2, p. 11-22, jul./dic. 2018. DOI:

http://dx.doi.org/10.15665/encuent.v16i02.974

VALLAEYS, F.; ÁLVAREZ, J. Hacia una definición latinoamericana de responsabilidad social universitaria. Aproximación a las preferencias conceptuales de los universitarios. Educación XX1, v. 22, n. 1, p. 93-116, 2019. Facultad de Educación. UNEDISSN: 1139613X. Disponible: http://revistas.uned.es/index.php/educacionXX1/article/view/19442/18458. Acceso: 10 dic. 2020.

VERA, K. El COVID-19, un asunto de salud y responsabilidad social. Pesquisa Javeriana, Artículo de opinión, 18 mar. 2020, Pontificia Universidad Javeriana-Colombia. Recuperado: https://www.javeriana.edu.co/pesquisa/el-covid-19-un-asunto-de-salud-y-responsabilidadsocial/. Acceso: 10 dic. 2020

\section{Cómo referenciar este artículo}

ROJAS, O.; MARTÍNEZ, M.; VIVAS, A. Responsabilidad social universitaria en tiempos de pandemia: mirada desde la función docente (Universidad de Antofagasta- Chile). Revista 
Ibero-Americana de Estudos em Educação, Araraquara, v. 16, n. 2, p. 424-439, abr./jun. 2021. e-ISSN: 1982-5587. DOI: https://doi.org/10.21723/riaee.v16i2.14707

Enviado: $30 / 11 / 2020$

Revisiones necesarias: $21 / 12 / 2020$

Aprobado: $26 / 01 / 2021$

Publicado: 01/02/2021 